\title{
Utility of an inflammation-based prognostic score for survival in oropharyngeal squamous cell carcinoma
}

\author{
Hiroyuki Iuchi*, Takayuki Kyutoku and Junichiro Ohori \\ Department of Otolaryngology, Head and Neck Surgery, Kagoshima University Graduate School of Medical and Dental Sciences, Kagoshima, Kagoshima, Japan
}

\begin{abstract}
Background: Several inflammation-based prognostic scoring systems have been devised and found to be strongly correlated with prognosis in patients with a variety of neoplasms.
\end{abstract}

Objectives: To investigate possible prognostic factors for patients with oropharyngeal squamous cell carcinoma (OSCC).

Material and methods: We retrospectively reviewed a database containing 68 patients who were diagnosed histologically with OSCC and who underwent surgery, radiotherapy, or chemo radiotherapy in our institute between March 2009 and May 2018. Prognosis was evaluated using overall survival (OS) and disease-free survival (DFS).

Results: Kaplan-Meier survival analysis revealed improved OS rates in patients with a modified Glasgow Prognostic Score (mGPS) of 0. Kaplan-Meier survival analysis also identified improved DFS rates in patients with human papillomavirus positivity, $\mathrm{N}$ classifications of 0 or 1 , and an mGPS of 0 or 1 . In our multivariate analysis, the survival benefit of the mGPS was independent of other factors.

Conclusions and significance: The results of this study confirmed that the mGPS is a good utility prognostic factor in patients with OSCC and may an important of detailed prognostic factor.

\section{Introduction}

The association of human papillomavirus (HPV) with head and neck cancer has been described, especially for oropharyngeal cancer (OPC) of the tonsils and tongue base, and the incidence of HPVpositive OPC is rapidly increasing in Europe and the United States [1]. HPV-related OPC is biologically different from OPC caused by conventional smoking and alcohol drinking, and good treatment outcomes have been achieved because chemotherapy and radiotherapy (RT) are effective [2]. Therefore, the TNM classification was revised in 2017 (UICC 8th Edition), and HPV-related OPC was classified as an independent item [3]. Furthermore, prognostic group classification using a prognostic factor grid was introduced as a new attempt. Among the factors considered prognostic for OPC, HPV positivity and the TN classification are associated with characteristics of the tumor and smoking and the performance score are associated with patient outcomes [3].

Several inflammation-based prognostic scoring systems have been devised and found to be strongly correlated with prognosis in patients with a variety of neoplasms. McMillan proposed the Glasgow Prognostic Score (GPS), an indicator of nutritional status based on inflammation and C-reactive protein (CRP) and albumin levels, and the score was identified as a prognostic factor independent of the stage of cancer [4]. Subsequently, it has been reported that the GPS is a prognostic factor in gastrointestinal cancer in addition to lung and gastric cancers $[5,6]$. However, no inflammation-based prognostic scoring systems have been described for OPC.

In the present study, we evaluated the clinical value of HPV, N classification, and the GPS in oropharyngeal squamous cell carcinoma (OSCC).

\section{Materials and methods}

\section{Patients}

This study was approved by the Institutional Review Board of Kagoshima University Graduate School of Medical and Dental Sciences. In total, 68 patients with OSCC who were treated in our institution from January 2010 to October 2018 were enrolled in this retrospective cohort study. The exclusion criteria were as follows: 1) duplicated cancer cases, 2) recurrent cancer, and 3) distant metastasis. The tumor HPV status was determined using p16 immunostaining and HPV DNA detection using PCR. All tests for the tumor HPV status were prospectively performed using untreated primary tumor tissue. Diffuse (at least more than 70\%) and strong nuclear and cytoplasmic staining of p16 or the detection of high-risk HPV DNA (HPV 16, 18, $33,35,39,45,51,52,56$, or 66 ) indicated positivity for HPV.

Docetaxel $60 \mathrm{mg} / \mathrm{m}^{2}$ (day 1$)+\operatorname{CDDP} 60 \mathrm{mg} / \mathrm{m}^{2}$ (day 1$)+5 \mathrm{FU}$ $600 \mathrm{mg} / \mathrm{m}^{2}$ (days 1-4) was administered as induction chemotherapy. Concurrent chemo radiotherapy (CCRT), consisting of carboplatin 80 $\mathrm{mg} / \mathrm{m}^{2}$ every week or cisplatin $100 \mathrm{mg} / \mathrm{m}^{2}$ every 3 weeks, was subsequently

${ }^{\star}$ Correspondence to: Hiroyuki Iuchi, Department of Otolaryngology, Head and Neck Surgery, Kagoshima University Graduate School of Medical and Dental Sciences, Kagoshima, Kagoshima, Japan, Tel: +81-99-275-5410; E-mail: hamoshun@m.kufm.kagoshima-u.ac.jp

Key words: oropharyngeal squamous cell carcinoma, modified Glasgow Prognostic Score, HPV, N classification

Received: April 30, 2020; Accepted: May 12, 2020; Published: May 15, 2020 
administered. All patients underwent computed tomography-based RT planning with either three-dimensional conformal RT or intensitymodulated RT. The patients' clinical characteristics, laboratory data, and treatment schedule were obtained from a retrospective review of their records. The pathological classification of the primary tumor, the degree of lymph node involvement, and the presence of organ metastasis were determined according to the TNM classification system (TNM Classification of Malignant Tumors, Eighth Edition) [7]. The patients' clinical characteristics, laboratory data, treatment, and were obtained from a retrospective review of the records.

Prognosis was evaluated using overall survival (OS) and diseasefree survival (DFS). OS was calculated from the date of diagnosis to that of death, and DFS was defined as survival from the date of diagnosis to that of the first detection of any recurrence (loco regional recurrence or distant metastasis), disease progression, or death.

\section{Inflammation-based prognostic scores}

Laboratory measurements, including the serum levels of CRP and albumin, were performed on the day of admission. GPS was calculated on the basis of these clinical data, and the modified GPS (mGPS) was constructed as previously described [8]. In the mGPS, patients exhibiting an elevated CRP level $(0.5 \mathrm{mg} / \mathrm{dL})$ and hypoalbuminemia $(3.5 \mathrm{~g} / \mathrm{dL})$ were given an mGPS of 2 , those with only one of these biochemical abnormalities were given an mGPS of 1 , and those with neither of these abnormalities were given an mGPS of 0 .

\section{Statistical analysis}

All statistical analyses were performed using JMP ${ }^{\circ} 14$. The KaplanMeier method was used to analyze survival, and the log-rank test was used to examine the differences in survival between the study groups. All $P$-values were two-sided. $P<0.05$ was considered statistically significant.

\section{Results}

\section{Demographic characteristics of the patients}

The baseline characteristic of the study patients is shown in table 1. Among the 68 patients with OSCC who underwent surgery or CCRT during the study period, the age at diagnosis ranged from 41 to 85 years (median, 65 years), and the dominant gender was male (61, 90\%). Thirty-one (45.6\%) patients had HPV-positive lesions. In total, $46(67.6 \%), 16(23.5 \%)$, and $6(8.9 \%)$ patients had mGPSs of 0,1 , and 2 , respectively. Other patient characteristics are summarized in table 1 .

\section{Associations of HPV, N classification, and the mGPS with OS}

Kaplan-Meier curves for OS for the entire cohort are shown in figure 1. The 5-year OS rates for patients with HPV-positive and HPVnegative lesions were 62.2 and $72.3 \%$, respectively. The 5 -year OS rates were $80.0,79.4$, and $36.1 \%$ for patients with N0, N1, and N2-3 tumors, respectively. The 5 -year OS rates were $83.2,0$, and $0 \%$ for patients with mGPSs of 0,1 , and 2, respectively. A significant association with OS was observed for the mGPS ( $\mathrm{p}=0.00009$ ) but not HPV positivity or the $\mathrm{N}$ classification. As shown in table 2, multivariate analysis including the aforementioned parameters revealed that the mGPS was an independent predictor of OS ( $\mathrm{p}<0.05)$.

\section{Associations of HPV, N classification, and the mGPS with DFS}

Kaplan-Meier curves for DFS for the entire cohort are presented in figure 2. The 5-year DFS rates where was 80.6 and $42.2 \%$ for patients
Table 1. Baseline patient characteristics $(n=68)$

\begin{tabular}{|c|c|}
\hline Variables & \\
\hline Age (median) & $41-85(65)$ \\
\hline \multicolumn{2}{|l|}{ Gender } \\
\hline Male & 61 \\
\hline Female & 7 \\
\hline \multicolumn{2}{|l|}{ HPV } \\
\hline Positive & 31 \\
\hline Negative & 37 \\
\hline \multicolumn{2}{|l|}{ Depth of tumor } \\
\hline T1 & 6 \\
\hline $\mathrm{T} 2$ & 29 \\
\hline T3 & 13 \\
\hline T4 & 20 \\
\hline \multicolumn{2}{|c|}{ Lymph node metastasis } \\
\hline No & 15 \\
\hline N1 & 25 \\
\hline $\mathrm{N} 2-3$ & 28 \\
\hline \multicolumn{2}{|l|}{ TNM stage } \\
\hline I & 20 \\
\hline II & 11 \\
\hline III & 10 \\
\hline IV & 27 \\
\hline \multicolumn{2}{|l|}{ mGPS } \\
\hline 0 & 46 \\
\hline 1 & 16 \\
\hline 2 & 6 \\
\hline \multicolumn{2}{|l|}{ Tumor location } \\
\hline Tonsil & 51 \\
\hline Base of the tongue & 13 \\
\hline Soft plate & 4 \\
\hline \multicolumn{2}{|l|}{ Treatment } \\
\hline NAC & 43 \\
\hline CCRT & 53 \\
\hline Surgery & 15 \\
\hline
\end{tabular}

NAC, neoadjuvant chemotherapy; CCRT, concurrent chemo radiotherapy; mGPS, modified Glasgow Prognostic Score

Table 2. Multivariate analysis of overall survival

\begin{tabular}{|l|c|c|}
\hline & \multicolumn{2}{|c|}{ Multivariate analysis } \\
\hline Variable & $\mathrm{p}$ & $\mathrm{HR}(95 \% \mathrm{CI})$ \\
\hline Alcohol & 0.53 & $1.89(0.26-13.64)$ \\
\hline $\mathrm{T}(1 / 2 / 3 / 4)$ & 0.22 & $1.84(0.69-4.87)$ \\
\hline $\mathrm{N}(0 / 1 / 2 / 3)$ & 0.29 & $0.29(0.03-2.79)$ \\
\hline Stage (I/II/III/IV) & 0.60 & $0.69(0.17-2.82)$ \\
\hline HPV (posi/nega) & 0.72 & $1.68(0.09-28.37)$ \\
\hline mGPS (0/1/2) & 0.0003 & $5.51(2.18-13.86)$ \\
\hline
\end{tabular}

HPV: human papillomavirus; posi: positive; nega: negative; mGPS: modified Glasgow Prognostic Score; HPV: human papillomavirus

Table 3. Multivariate analysis of disease-free survival

\begin{tabular}{|l|c|c|}
\hline & \multicolumn{2}{|c|}{ Multivariate analysis } \\
\hline Variable & p & HR (95\% CI) \\
\hline Alcohol & 0.25 & $0.51(0.16-1.62)$ \\
\hline $\mathrm{T}(1 / 2 / 3 / 4)$ & 0.17 & $1.59(0.82-3.08)$ \\
\hline $\mathrm{N}(0 / 1 / 2 / 3)$ & 0.079 & $5.39(0.82-35.33)$ \\
\hline Stage (I/II/III/IV) & 0.60 & $0.61(0.19-1.93)$ \\
\hline HPV (posi/nega) & 0.31 & $0.31(0.03-2.94)$ \\
\hline mGPS (0/1/2) & 0.016 & $2.02(1.14-3.56)$ \\
\hline
\end{tabular}

HPV: human papillomavirus; posi: positive; nega: negative; mGPS: modified Glasgow Prognostic Score; HPV: human papillomavirus 

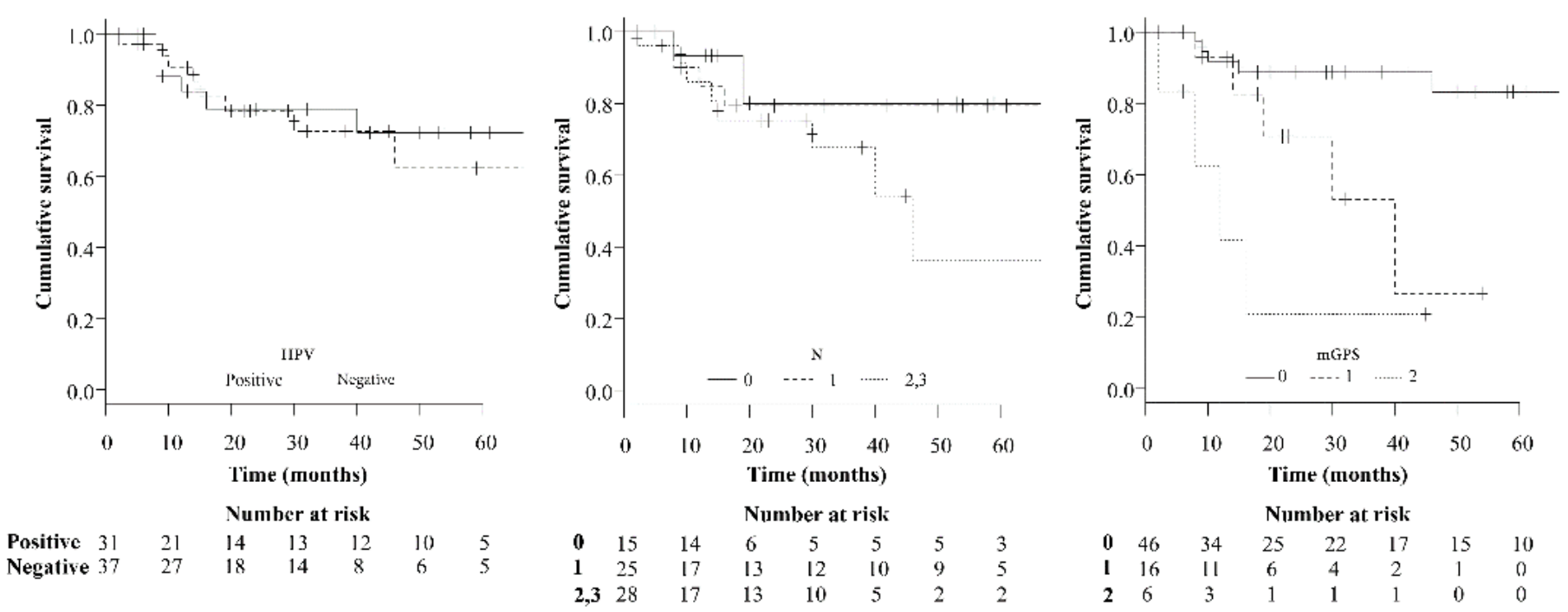

Figure 1. Overall survival of patients according to HPV positivity, N classification, and mGPS, modified Glasgow Prognostic Score; HPV, human papillomavirus.
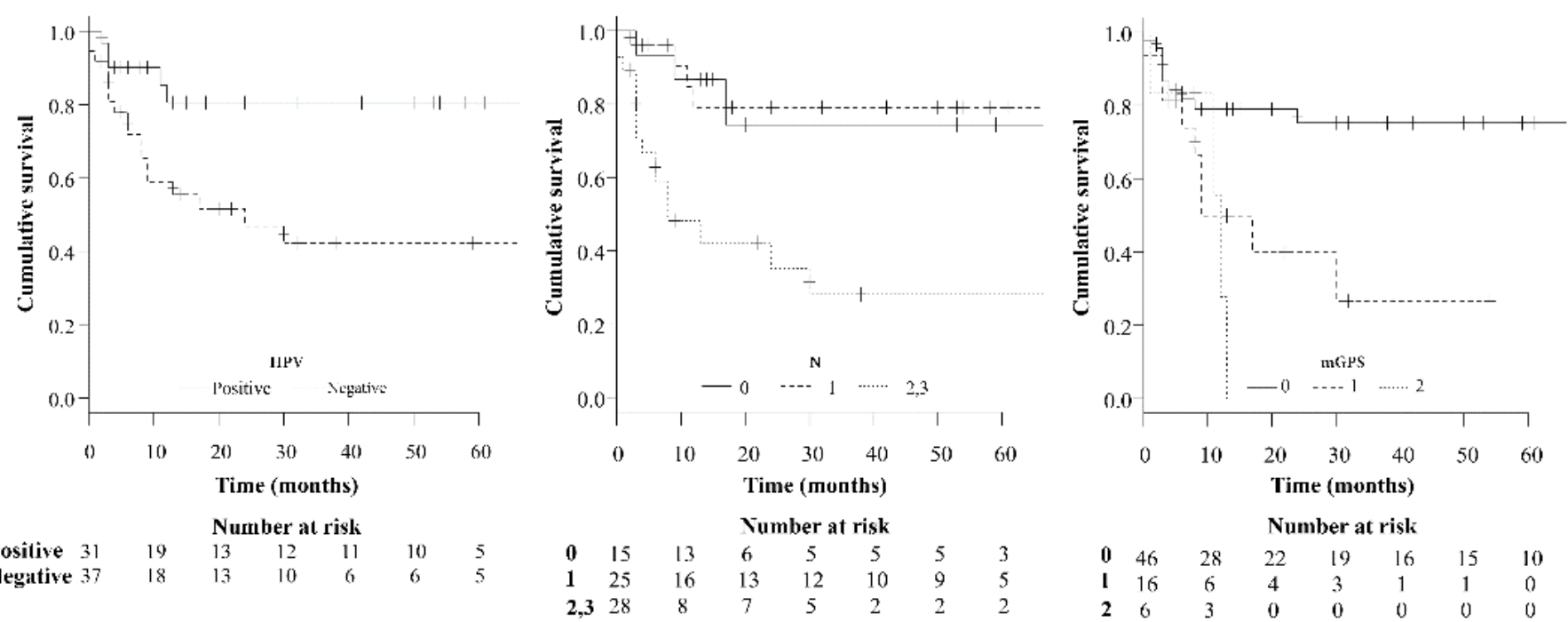

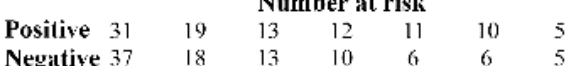

$\begin{array}{llcccccc}\mathbf{0} & 15 & 13 & 6 & 5 & 5 & 5 & 3 \\ \mathbf{1} & 25 & 16 & 13 & 12 & 10 & 9 & 5 \\ \mathbf{2 , 3} & 28 & 8 & 7 & 5 & 2 & 2 & 2\end{array}$

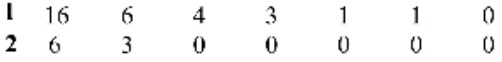

Figure 2. Disease-free survival of patients according to HPV positivity, N classification, and mGPS mGPS, modified Glasgow Prognostic Score; HPV, human papillomavirus.

with HPV-positive and HPV-negative tumors, respectively. The 5-year DFS rates were $74.3,79.1$, and $28.1 \%$ for patients with $\mathrm{N} 0, \mathrm{~N} 1$, and $\mathrm{N} 2-3$ tumors. The overall 5-year DFS rates were $75.2,0$, and $0 \%$ for patients with mGPSs of 0,1 , and 2 , respectively. HPV positivity ( $\mathrm{p}=$ $0.009)$, $\mathrm{N}$ classification $(\mathrm{p}=0.0003)$, and the mGPS ( $\mathrm{p}=0.006)$ were demonstrated to be significantly associated with DFS. As shown in table 3 , multivariate analysis including the aforementioned parameters revealed that only mGPS was an independent predictor of DFS $(\mathrm{p}<0.05)$.

\section{Discussion}

As a mechanism of carcinogenesis induced by HPV, the basal cells of the squamous layer are first infected through mucosal wounds, and viral DNA is maintained as nuclear episomes of the basal cells $[8,9]$. Most HPV-related OSCCs occur at sites including tonsillar tissue such as palatine tonsils and the base of the tongue [10]. In this examination, lesions arising in palatine tonsils and tongue roots accounted for $64 / 68$ cases $(94 \%)$. It has been revealed that palatine tonsils and the tongue base have high affinity for HPV [11]. In this study, HPV was detected in 30/53 cases (56.6\%) submitted for examination, most frequently HPV 16, in line with the results of previous research [12]. When the HPV genome is integrated into the host chromosome, it is believed that constitutive overexpression of E6 and E7 facilitates the acquisition of malignant traits through the functions of E6 and E7 oncoproteins [13]. Because p16, one of the cyclin-dependent kinases, is highly expressed as a negative feedback against accelerated cell cycle turnover, p16 is recognized as a surrogate marker for HPV. The cancer was roughly divided into positive and negative types based on the p16 immunohistological examination. p16 immunostaining can serve as a surrogate marker for HPV positivity because when HPV infects a cell, HPV E7 protein binds to Rb, leading to the dissociation of E2F, and the subsequent increase of $\mathrm{p} 16$ expression prevents cells from progressing to $S$ phase [14]. In our department, p16 immunohistological examination has been conducted since 2018. In this study, HPV was considered positive for cases in which HPV DNA was detected by PCR or p16 positivity was confirmed, whereas other cases were considered negative. When comparing the 5-year survival rates between the HPV-positive 
and HPV-negative cohorts, a statistically significant difference was identified only for DFS. The cause of no difference in OS is considered to be the cause of many death cases among other HPV-positive cases.

HPV-related cancers have a higher degree of $\mathrm{N}$ progression even among relatively early $\mathrm{T}$ cases compared with the finding for nonrelated cancers, and a higher rate of progression is observed in advanced cancers. In p16-positive OPC in the new TNM classification, the clinical N classification was largely changed, and N1 to N2b of the old TNM classification (7th edition) was concentrated into N1. In addition, $\mathrm{T} 4 \mathrm{a}$ and $\mathrm{T} 4 \mathrm{~b}$ were condensed to $\mathrm{T} 4$ in the $\mathrm{T}$ classification without a distinction between then. Therefore, because there were many changes in the $\mathrm{N}$ classification, this classification was examined in the present study. Regarding this classification, cases arising before the TNM classification revision were classified and examined retrospectively. In addition, because there were only three patients with $\mathrm{N} 3$ tumors, they were examined together with patients with N2 tumors. Similarly, as observed for HPV, only DFS was significantly different according to the $\mathrm{N}$ classification. In other words, we believe that further study of prognostic factors is necessary for OS.

Thus, we focused on GPS. The inflammation-based GPS is a scoring system based on elevated CRP serum levels and hypoalbuminemia [15]. Elevated serum CRP levels reflect a state of systemic inflammation, and this finding is generally associated with a high cancer risk and poor prognosis [16]. Hypoalbuminemia reflects the hyper catabolic state of cancer cachexia caused by activated cytokines, and it is commonly observed in patients with cancer [17]. In the present study, the mGPS was used to reflect these variables. The present study revealed that the mGPS is an excellent prognostic factor for both OS and DFS. Furthermore, multivariate analysis confirmed that the mGPS is an independent predictor of survival. This indicates that GPS is a scoring system that shows bias in acute phase proteins (APPs) dynamics, that is, independent of the clinical stage of cancer. The mGPS is considered to indirectly indicate the existence of "cancer cachexia" [18].

Some limitations of the study should be acknowledged. First, this was a retrospective study, conducted at a single institute without blindness or control groups. Second, the number of patients studied was small. In the future, larger prospective studies are required to establish the significance of the GPS in OSCCs.

\section{Conclusion}

In this study, the mGPS, but not HPV positivity or the $\mathrm{N}$ classification, was identified as an independent prognostic factor in OS and DFS for patients with OSCC.

\section{Reference}

1. Nasman A, Attner P, Hammarstedt L, Du J, Eriksson M, et al. (2009) Incident of human papillomavirus (HPV) positive tonsillar carcinoma in Stockholm, Sweden: an epidemic of vira-induced carcinoma? Int J Cancer 125: 362-366. [Crossref]

2. Ang KK, Harris J, Wheeler R, Weber R, Rosenthal DI, et al. (2010) Human papillomavirus and survival of patients with oropharyngeal cancer. $N$ Engl J Med 363: 24-35. [Crossref]

3. Amin MB, Edge SB, Greene FL, (2017) American Joint Committee on Cancer (AJCC) Cancer Staging Manual. ( $8^{\text {th }}$ edn,) Springer, New York, pp: 55-181.

4. McMillan DC (2008) An inflammation-based prognostic score and its role in the nutrition-based management of patients with cancer. Proc Nutr Soc 67 :257-262. [Crossref]

5. Forrest LM, McMillan DC, McArdle CS, Dunlop DJ (2003) Evaluation of cumulative prognostic scores based on the systemic inflammatory response in patients with inoperable non-small-cell lung cancer. Br J Cancer 89:1028-1030. [Crossref]

6. Elahi MM, McMillan DC, McArdle CS, Angerson WJ, Sattar N (2004) Score based on hypoalbuminemia and elevated C-reactive protein predicts survival in patients with advanced gastrointestinal cancer. Nutr Cancer 48: 171-173. [Crossref]

7. James DB, Mary KG, Christian W (2017) International Union against Cancer (UICC). TNM classification of malignant tumours. $\left(8^{\text {th }}\right.$ edn), Wiley, New York.

8. Ye LL, Oei RW, Kong FF, Du CR, Zhai RP, et al (2018) The prognostic value of preoperative prognostic nutritional index in patients with hypopharyngeal squamous cell carcinoma: A retrospective study. J Transl Med 16: 12. [Crossref]

9. Kato H, Naito K (2016) Oropharyngeal cancer and HPV. Practica Otologica Pract 109: $371-381$.

10. Shi W, Kato H, Perez-Ordonez B, Pintilie M, Huang S, et al. (2009) Comparative prognostic value of HPV16 E6 mRNA compared with in situ hybridization for human oropharyngeal squamous carcinoma. J Clin Oncol 27: 6213-6221. [Crossref]

11. Hobbs CG, Sterne JA, Bailey M, Heyderman RS, Birchall MA, et al. (2006) Human papillomavirus and head and neck cancer: a systematic review and metaanalysis. Clin Otolaryngol 31: 259-266. [Crossref]

12. Hama T, Tokumaru Y, Fujii M, Yane K, Okami K, et al. (2014) Prevalence of human papillomavirus in oropharyngeal cancer: a multicenter study in Japan. Oncology 87: 173-182. [Crossref]

13. Minaguchi $\mathrm{T} \square$ Yoshikawa $\mathrm{H} \quad$ (2010) Molecular mechanism of cervical carcinogenesis $\square$ Jpn J Cancer Chemother 37: 18-22.

14. Yane K (2018) Revision and problems of new TNM classification. Oropharyngeal Cancer 44: 253-257.

15. Al Murri AM, Bartlett JM, Canney PA, Doughty JC, Wilson C, et al. (2006) Evaluation of an inflammation-based prognostic score (GPS) in patients with metastatic breast cancer. Br J Cancer 94: 227-230. [Crossref]

16. Allin KH, Bojesen SE, Nordestgaard BG (2009) Baseline C-reactive protein is associated with incident cancer and survival in patients with cancer. J Clin Oncol 27 : 2217-2224. [Crossref]

17. Teunissen SC, Wesker W, Kruitwagen C, de Haes HCJM, Voest EE, et al. (2007) Symptom prevalence in patients with incurable cancer: a systematic review. J Pain Symptom Manage 34: 94-104. [Crossref]

18. McMillan DC (2009) Systemic inflammation, nutritional status and survival in patients with cancer. Curr Opin Clin Nutr Metab Care 12: 223-226. [Crossref]

Copyright: (C2020 Iuchi H. This is an open-access article distributed under the terms of the Creative Commons Attribution License, which permits unrestricted use, distribution, and reproduction in any medium, provided the original author and source are credited. 